\title{
Lebensbedingungen im europäischen Langstrafenvollzug
}

Kirstin Drenkhahn und Manuela Dudeck

W eltweit steigt die Anzahl der Gefängnisinsassen dramatisch an. Allein zwischen Februar 2005 und Oktober 2006 war eine Zunahme von mehr als 250.000 Insassen zu verzeichnen. Insgesamt waren 2006 über 9,25 Millionen Menschen in Einrichtungen des Strafvollzugs untergebracht, in der EU waren es mehr als 600.000 (Walmsley 2006).

Da der Strafvollzug als Teil der Strafvollstreckung einer der Bereiche ist, die von der polizeilichen und justiziellen Zusammenarbeit in Strafsachen in der EU erfasst werden, wird die Union sich in absehbarer Zeit mit den Problemen ihrer Gefangenen auseinandersetzen müssen. Ebenso wie bei der Strafverfolgung führen auch hier die nationalen Besonderheiten der Mitgliedstaaten zu Schwierigkeiten. Zwar hielt die Europäische Kommission in ihrer Mitteilung an den Rat und das Europäische Parlament vom 19.5.2005 (KOM (2005) 195 endg) fest, dass eine strafrechtliche Verurteilung, die in einem Mitgliedstaat ergangen ist, auch in jedem anderen Mitgliedstaat vollstreckt werden können muss. Allerdings konzentrieren sich die Bemühungen im Rahmen der justiziellen Zusammenarbeit in Strafsachen bisher auf das materielle Strafrecht und das Strafprozessrecht, der Strafvollzug war bisher nur Gegenstand einer Empfehlung des Europäischen Parlaments an den Rat (P5 TA(2004)0142 vom 9.3.2004).

Insbesondere der Vollzug freiheitsentziehender Sanktionen wirft im Hinblick auf zwei der zentralen Werte der EU, Freiheit und soziale Gerechtigkeit, Fragen auf. Zunächst bestimmt nicht nur die Länge einer Sanktion, sondern es bestimmen auch die Bedingungen ihrer Vollstreckung ihre tatsächliche Schwere und damit die Reichweite des Freiheitsentzugs. Darüber hinaus haben die Vollzugsbedingungen maßgeblichen Einfluss auf die Legalbewährung eines Gefangenen und seine Chancen auf soziale Teilhabe nach seiner Entlassung in die Freiheit wie z. B. die Reintegration in den Arbeitsmarkt. Außerdem stellt sich bei der Vollstreckung freiheitsentziehender Sanktionen immer das Problem, inwieweit die Betroffenen ihre Grundrechte wahrnehmen können. Die besondere Bedeutung der Grundrechte in der EU zeigt sich z. B. dadurch, dass ihre Stärkung eine der zehn Prioritäten des Haager Programms ist, das der Europäische Rat im November 2004 angenommen hat.

Der Vollzug langer Freiheitsstrafen birgt im Hinblick auf die Menschenrechtssituation besondere Risiken. Als lang werden hier entsprechend der Empfehlung des Europarats zum Vollzug langer Freiheitsstrafen (Rec(2003)23 vom 9.10.2003) lebenslange Freiheitsstrafen und solche von mindestens fünf Jahren bezeichnet. Diese Gefangenen sind zumeist wegen schwerer Straftaten inhaftiert und gelten häufig als besonders gefährlich. Im Anstaltsalltag kann sich dies auswirken, indem Gefangene grundsätzlich nicht zu Rehabilitationsmaßnahmen zugelassen werden, da dies aufgrund der Straflänge und der Gefährlichkeit zwecklos erscheint. Hinzu kommen die negativen Auswirkungen des Freiheitsentzugs in Form von Haftdeprivationen, die grundsätzlich jeder Inhaftierung innewohnen, sich jedoch bei besonders langem Freiheitsentzug verstärkt bemerkbar machen. Ein weiteres Problem ist die psychische Gesundheit der Gefangenen. Vor dem Hintergrund des steigenden gesellschaftlichen Sicherheitsbedürfnisses dürften die Aufnahmen von psychisch Kranken in Gefängnisse erheblich an Bedeutung gewinnen und auch therapeutische Implikationen haben. Die Häufigkeit psychischer Störungsbilder im Strafvollzug wächst stetig an und ist damit im Vergleich zur Allgemeinbevölkerung deutlich erhöht (Blocher et al. 2001). Momentan ist diese Klientel sicherlich unzureichend untersucht und behandelt, obwohl für Häftlinge der gleiche gesundheitliche Versorgungsstandard wie für die Allgemeinbevölkerung gelten soll (Empfehlung des Europarats Rec(2006)2 - European Prison Rules, Nr. 40.2; Andersen 2004; Wilson 2004; Birmingham et al. 2006). Je nach Schwere können psychische Erkrankungen erhebliche Auswirkungen auf die Bewältigung des Alltags haben. In ca. $25 \%$ der Fälle kommt es zum Opfer/Täter Transfer; viele Insassen erleben neu in Haft entstehende Gewalt und entwickeln so Traumafolgestörungen wie die Posttraumatische Belastungsstörung (PTSD) (Spitzer et al. 2006; Dudeck et al. 2007). Des Weiteren spielt die Induktion vorher latent vorhandener Störungen wie die soziale Phobie eine wichtige Rolle (Dudeck/Freyberger in press).

Somit besteht also die Gefahr, dass sich bei Personen, die nach langem Freiheitsentzug entlassen werden, die Schwierigkeiten bei der Wiedereingliederung in das Leben in Freiheit verglichen mit anderen Haftentlassenen potenzieren. Werden für Langzeitinhaftierte Behandlungs- und Betreuungsmaßnahmen angeboten, können Probleme im Hinblick auf eine kontinuierliche Betreuung über die Zeit im Vollzug hinaus nach der Entlassung bestehen. Im Folgenden soll versucht werden, die Bedeutung des Langstrafenvollzugs in den EUMitgliedstaaten sowie die psychische Gesundheit und die Lebensbedingungen der Gefangenen zu beschreiben.

\section{Quantitative Bedeutung langer Freiheits- strafen im Strafvollzug der EU-Mitglieder}

Die EU selbst erstellt keine Statistiken zum Strafvollzug in ihren Mitgliedstaaten. Allerdings sind alle EU-Staaten auch Mitglied des Europarats, der eine jährliche Statistik - SPACE I - zum Strafvollzug herausgibt. An der Erhebung für 2005 haben sich alle EU-Mitgliedstaaten außer der Republik Irland beteiligt (SPACE I 2005, S. 2). Detaillierte Daten zu den Straflängen lagen nicht vor von Bulgarien, Ungarn und Zypern. Bei der Betrachtung dieser Daten muss man sich der Tatsache bewusst sein, dass hier nationale Statistiken zusammengetragen wurden, in denen zum Teil andere Kategorien verwendet werden als bei SPACE I. Außerdem sind die Bearbeiter von dem Material abhängig, das ihnen die Mitgliedstaaten liefern. Trotzdem kann man festhalten, dass Freiheitsstrafen von mindestens fünf Jahren im Strafvollzug der einzelnen Mitgliedstaaten einen ganz unterschiedlichen Stellenwert haben. Extrembeispiele sind Griechenland mit ca. 70\% Langzeitgefangenen und Finnland mit ca. $13 \%$. Insgesamt lassen sich hier drei Gruppen entsprechend der Anteile von Langzeitgefangenen einteilen: Eine Gruppe mit einem geringen Anteil von weniger als 20\% Langzeitgefangenen mit Dänemark, Deutschland, Finnland, den Niederlanden, Polen und der Tschechischen Republik, eine Gruppe mit 
einem sehr hohen Anteil von mehr als 50\% mit Belgien, Griechenland und Portugal und ein starkes Mittelfeld mit Anteilen zwischen $20 \%$ und $50 \%$. Warum es diese großen Unterschiede gibt, lässt sich anhand der SPACE-Daten nicht klären. Dazu wären Informationen über die Strafrahmen und die Verurteilungspraxis sowie über die Deliktsverteilung im Strafvollzug mit Blick auf die Straflänge erforderlich (vgl. zum ähnlichen Problem der Erklärung unterschiedlicher Gefangenenraten von Hofer 2004). Zwar enthält SPACE I auch Daten zur Deliktsverteilung, allerdings nicht auf die Straflänge bezogen. Darüber hinaus ist aus den Kommentaren zu den Daten über die Deliktsverteilung zu ersehen, dass es Probleme aufwirft, die Deliktskategorien einheitlich zu definieren (vgl. SPACE I 2005, S. 35 ff.). Die Anzahl der Langzeitgefangenen hat sich in den EU-Staaten in den letzten Jahren eher - teilweise erheblich - vergrößert (z. B. Belgien, England und Wales, Niederlande, Italien, Schweden) oder ist geschwankt; nur in wenigen Staaten ist die Gruppe kontinuierlich kleiner geworden (z. B. Estland, Finnland, vgl. dazu die SPACE I-Berichte 2000-2005).

Daten zur Sozial- und Legalbiographie enthalten die SPACE I-Berichte nicht. Andere Statistiken auf europäischer Ebene, die derartige Informationen beinhalten, gibt es nicht, so dass eine genauere Beschreibung der Gruppe der Langzeitgefangenen anhand international einheitlicher Kriterien vorerst nicht möglich ist.

\section{Psychische Gesundheit}

Umfassende Untersuchungen zur psychischen Gesundheit von Langzeitinhaftierten in Europa scheint es bisher nicht zu geben. Daher soll hier auf Daten zur allgemeinen Strafvollzugspopulation zurückgegriffen werden.

Bereits inhaftierte Jungen im Alter von 12 bis 18 Jahren weisen vermehrt psychopathologische Phänomene auf. Führend ist dabei die Störung des Sozialverhaltens (75\%), gefolgt von der Substanzabhängigkeit (55\%) und psychotischen Symptomen (34\%). Affektive Syndrome (6\%) und Angststörungen (9\%) spielen ebenfalls eine nicht unwesentliche Rolle (Vreugdenhil et al. 2004). Ganz ähnliche Befunde konnten Jacobs/Reinhold (2004) bei Jugendlichen von 18 bis 23 Jahren finden, wobei besonders die Lebenszeitprävalenz von $63,5 \%$ für die substanzbezogenen Störungen auffällig war. Mit $65 \%$ hatte die Mehrheit der Probanden schon eine Persönlichkeitsstörung entwickelt, davon 52\% eine antisoziale Persönlichkeitsstörung, die sich mit hoher Wahrscheinlichkeit aus der Störung des Sozialverhaltens ausgebildet hat. Die Prävalenz des Aufmerksamkeitsdefizit-/Hyperaktivitätssyndroms (ADHS) bei jugendlichen Insassen rangiert bei $8 \%$ bis $45 \%$ (Vreugdenhil et al. 2004; Rasmussen et al. 2001; Rösler et al. 2004). Zusammenfassend haben bis zu $60 \%$ der inhaftierten Jugendlichen mindestens eine relevante psychische Störung (Jacobs/Reinhold 2004).
Im Hinblick auf die erwachsenen Inhaftierten ist von einer Prävalenz psychischer Störungen von 38-94\% auszugehen. Differierende Prävalenzwerte in den vorliegenden Studien werden sowohl durch spezifische Merkmale der untersuchten Population als auch durch die Erhebungsmethoden bestimmt. Daher sind länderübergreifende Verallgemeinerungen schwierig, führen häufig zu unscharfen Aussagen und zeigen eine große Spannweite (Roesch et al. 1995, Konrad 2000). Die nebenstehende Tabelle zeigt die aktuellen Arbeiten im europäischen Raum.

Ältere Inhaftierte, d. h. 60jährige und Ältere, weisen ebenfalls zu 5\% psychotische Störungen auf. Die Affektiven Störungen, insbesondere Depressionen, machen etwa ein Viertel der Erkrankungen aus. Bei 30\% bestehen Persönlichkeitsstörungen. Im Vergleich zu jugendlichen und erwachsenen Inhaftierten sinkt die Zahl der Substanzabhängigen unter $5 \%$ (Fazel et al. 2001).

Aus der hohen Prävalenz psychischer Symptome über alle Altersgruppen hinweg ergeben sich für die Planung und Durchführung der Haft immer wieder weit reichende Konsequenzen wie z. B. die Verlegung in eine psychiatrische Abteilung innerhalb der Justizvollzugsanstalten oder kurz- und mittelfristige Isolierungen oder Überwachungen (Cooke/Mitchie 1996).

Tabelle 1: Prävalenz psychischer Erkrankungen bei männlichen Gefangenen in Europa

\begin{tabular}{|c|c|c|c|c|c|c|c|}
\hline Autor & Land & $\mathbf{N}$ & $\begin{array}{l}\text { Abhängigkeit } \\
\text { von Alkohol } \\
\text { und Drogen }\end{array}$ & $\begin{array}{l}\text { Psychotische } \\
\text { Störungen }\end{array}$ & $\begin{array}{l}\text { Affektive } \\
\text { Störungen }\end{array}$ & $\begin{array}{l}\text { Angst- } \\
\text { störungen }\end{array}$ & $\begin{array}{l}\text { Persönlich- } \\
\text { keitsstörun- } \\
\text { gen }\end{array}$ \\
\hline Andersen et al., 1996 & DK & $228^{*}$ & $44 \%$ & $7 \%$ & $10 \%$ & $16 \%$ & $17 \%$ \\
\hline Birmingham et al., 1996 & GB & $569^{*}$ & - & $24 \%$ & $27 \%$ & $34 \%$ & $38 \%$ \\
\hline Brooke et al., 1996 & GB & $750^{*}$ & $38 \%$ & $5 \%$ & $22 \%$ & $18 \%$ & $11 \%$ \\
\hline Carrá et al., 2004 & IT & 990 & $47 \%$ & $1 \%$ & $5 \%$ & $2 \%$ & $4 \%$ \\
\hline Falissard et al., 2006 & FR & 800 & $14 \%$ & $12 \%$ & $21 \%$ & $21 \%$ & - \\
\hline Farrell et al., 2002 & GB & 503 & $29 \%$ & $10 \%$ & - & - & - \\
\hline Fotiadou et al., 2006 & GR & $80^{*}$ & $53 \%$ & $11 \%$ & $28 \%$ & $38 \%$ & $38 \%$ \\
\hline Harsch et al., 2006 & $\mathrm{DE}$ & 56 & $68 \%$ & - & $11 \%$ & $3 \%$ & $34 \%$ \\
\hline Joukamaa, 1995 & FI & 283 & $44 \%$ & $3 \%$ & - & $6 \%$ & $17 \%$ \\
\hline Konrad, 2004 & $\mathrm{DE}$ & $100^{* *}$ & $57 \%$ & $9 \%$ & $34 \%$ & $10 \%$ & - \\
\hline Langeveld et al., 2004 & $\mathrm{NO}$ & 40 & $90 \%$ & $32 \%$ & - & - & $80 \%$ \\
\hline Missoni et al., 2001 & DE & $108^{*}$ & $13 \%$ & $6 \%$ & $48 \%$ & $20 \%$ & - \\
\hline v. Schönfeld et al., 2006 & DE & 76 & $64 \%$ & $4 \%$ & $12 \%$ & $17 \%$ & $43 \%$ \\
\hline Watzke et al., 2006 & $\mathrm{DE}$ & 366 & $60 \%$ & $0,5 \%$ & $6 \%$ & $7 \%$ & $26 \%$ \\
\hline
\end{tabular}

*Untersuchungsgefangene, ${ }^{* *}$ Ersatzfreiheitsstrafe 
Dies zu vermeiden, macht die Präsenz einer suffizienten psychiatrischen Therapie vor Ort erforderlich, zumindest aber die Notversorgung von psychischen Ausnahmesituationen. Eine Verbesserung der psychischen Symptomatik bei einem Viertel der inhaftierten Erkrankten konnte bereits die Liaison Psychiatrie im Gefängnis Rottenburg (Deutschland) zeigen (Schafer et al. 2004). Der Fokus der psychosozialen Versorgungs- und Behandlungsmaßnahmen sollte in der multiprofessionellen Behandlung von Alkohol- und Drogenproblematiken liegen (Jacobs/Reinhold 2004). Betrachtet man die Häufigkeit der antisozialen Persönlichkeitsstörung, sind Psychoedukation, soziales Kompetenztraining und Training von Selbstmanagement sinnvoll (Kanfer et al. 2004; Hinsch/Pfingsten 2007). Nur so kann die Rückfallwahrscheinlichkeit gesenkt und die Wiedereingliederung von psychisch erkrankten Häftlingen verbessert werden.

\section{Forschung zu Lebensbedingungen Inhaftierter}

Forschung $\mathrm{zu}$ den Lebensbedingungen von Gefangenen in europäischen Strafvollzugseinrichtungen ist bisher nur spärlich vorhanden. Wenn Lebensbedingungen in Untersuchungen eine Rolle spielen, dann in der Regel eine untergeordnete; die Befunde bilden dann den Hintergrund für das eigentliche Thema des Projekts. So werden z. B. bei Untersuchungen zur Wirksamkeit sozialtherapeutischer Behandlung auch die Unterbringungssituation und die Organisation des alltäglichen Lebens beschrieben (Bussmann/Seifert 2002; Bussmann et al. 2004; Dünkel 1980; Ortmann 2002), da diese Rahmenbedingungen einen wesentlichen Teil des sozialtherapeutischen Konzepts ausmachen (vgl. Arbeitskreis 2001; Specht 2000).

Ein weiteres Beispiel ist die britische Forschung der letzten 15 Jahre. Der Schwerpunkt liegt auf der Untersuchung der sozialen Beziehungen im Strafvollzug als Grundlage der Legitimität der inneren Ordnung (vgl. dazu die Arbeiten von Bottoms, Hay und Sparks, z. B. Sparks/Bottoms/Hay 1996) und der moralischen Legitimität eines Gefängnisses (vgl. dazu die Arbeiten der Gruppe um Liebling, z. B. Liebling 2004). Die Unterbringungssituation, Versorgung z. B. mit Lebensmitteln, sauberer Kleidung und Bettwäsche, die Möglichkeit, Briefe zu schreiben und zu empfangen und Besuche $\mathrm{zu}$ erhalten und andere Lebensbereiche werden hier erhoben, um u. a. vor diesem Hintergrund die sozialen Beziehungen der Gefangenen untereinander und zwischen Gefangenen und Bediensteten analysieren zu können (Lieb- ling 2004, S. 260 ff.; Sparks/Bottoms/Hay 1996, S. $159 \mathrm{ff}$.$) .$

Für die Beschreibung der Lebensbedingungen im Strafvollzug bieten diese und andere Projekte Anhaltspunkte, allerdings lässt sich kein umfassendes Bild zeichnen. Die Befunde sind nicht miteinander vergleichbar, da die Daten nicht einheitlich erhoben wurden. Darüber hinaus bieten die Berichte nur einen Ausschnitt aus dem Strafvollzug eines bestimmten Landes. Gemeinsamkeiten und Unterschiede zwischen den Strafvollzugssystemen in Europa können so nicht sichtbar gemacht werden. Außerdem untersuchen gerade die Studien zur Sozialtherapie und Studien zu ähnlichen Vollzugsformen aus dem Ausland besondere, teilweise historische Vollzugssituationen, so dass die Darstellungen nicht verallgemeinert werden können.

\section{Berichte des Anti-Folter-Komitees}

Als weitere Quelle für Informationen zu den tatsächlichen Lebensumständen in Einrichtungen des Justizvollzugs kommen die Berichte des Europäischen Komitees zur Verhütung von Folter und unmenschlicher oder erniedrigender Behandlung oder Strafe (Anti-Folter-Komitee, CPT) über seine Besuche in den Mitgliedstaaten des Europarats in Frage. Bei diesen mehrtägigen Besuchen begehen die Mitglieder des CPT u. a. Strafvollzugsanstalten und unterhalten sich - ohne Zeugen - mit Gefangenen. Die Ergebnisse eines Besuchs werden zusammen mit daraus gefolgerten Empfehlungen in einem Bericht an den besuchten Staat festgehalten, der üblicherweise veröffentlicht wird (vgl. CPT 2003). Das Anti-Folter-Komitee hat auf der Grundlage der Berichte eigene Standards entwickelt, an denen die tatsächliche Situation der Gefangenen in den besuchten Einrichtungen gemessen wird (CPT 2004). Der Vollzug langer Freiheitsstrafen bzw. die Situation von Personen, die lange Zeit untergebracht sind wie z. B. auch Sicherungsverwahrte, ist einer der Bereiche, denen das Komitee besondere Aufmerksamkeit widmet. Das CPT hat folgende Formel entwickelt:

„Langzeithaft kann eine Reihe entsozialisierende Wirkungen auf die Insassen haben. Über das Institutionalisiertwerden hinaus können Langzeitgefangene viele psychische Probleme entwickeln (etwa Verlust der Selbstachtung und Abbau sozialer Fertigkeiten) sowie die Tendenz, sich immer mehr von der Gesellschaft abzulösen, in die die meisten von ihnen letztlich zurückkehren werden. Aus Sicht des CPT sollten die Haftregimes für Langzeitgefangene anstreben, diese Auswirkungen in posi- tiver und proaktiver Weise auszugleichen." (CPT 2004, S. 30).

Auf diese Formel folgen die folgenden allgemeinen Empfehlungen:

„Die betroffenen Gefangenen sollten Zugang zu einer großen Bandbreite sinnvoller Aktivitäten unterschiedlicher Art haben (Arbeit, vorzugsweise mit berufbezogenem Wert; Bildung; Sport; Erholung/Geselligkeit). Überdies sollten sie eine gewisse Auswahl über die Art und Weise, wie sie ihre Zeit verbringen wollen, ausüben können und so ein Gefühl für Autonomie und persönliche Verantwortung entwickeln. Zusätzliche Schritte sollten unternommen werden, um ihrer Haftzeit einen Sinn zu verleihen; insbesondere können diesen Gefangenen individualisierte Vollzugspläne und geeignete psychosoziale Unterstützung dabei helfen, mit der Dauer ihrer Inhaftierung zurechtzukommen und sich, wenn es soweit ist, auf die Entlassung vorzubereiten. Darüber hinaus ist die negative Wirkung der Institutionalisierung auf Langzeitgefangene weniger ausgeprägt und sie sind besser für die Entlassung gerüstet, wenn es ihnen möglich ist, nachhaltig Kontakt mit der Außenwelt zu bewahren." (CPT 2004, S. 30)

Eine Durchsicht der CPT-Berichte über Besuche in den derzeitigen EU-Mitgliedstaaten seit 1994 zeigt, dass die Situation von Langzeitinhaftierten - kaum überraschend - sehr unterschiedlich ist. Allerdings hängt das Maß, in dem die Lebensbedingungen den Vorgaben des Komitees entsprechen, nicht nur von der wirtschaftlichen Lage eines Staates ab.

Ein weit verbreitetes Problem ist die Einordnung von Langstrafern als per se gefährlich, ohne dass eine individuelle Risikoprognose erstellt wird. Das betrifft vor allem zu lebenslanger Freiheitsstrafe Verurteilte (vgl. die Berichte über die Besuche in Bulgarien 1999 , Estland 2003). Die Gefangenen sind in einer Hochsicherheitsabteilung untergebracht, meist allein oder zu zweit in einem Haftraum, den sie nur für die Freistunde und wenige andere Aktivitäten verlassen dürfen. Sie haben keinen oder nur sehr wenig Kontakt zu anderen Gefangenen (vgl. die Berichte über Bulgarien 1999, 2002, Estland 1997, Italien 1995 2004, Lettland 2002, Litauen 2004, Slowakei 1995, 2005, Tschechische Republik 1997, 2002, 2006), so dass teilweise auch die Freistunde alleine, zum Teil in dafür vorgesehenen Kabinen (cubicles), verbracht wird (vgl. die Berichte über Lettland 1999, Tschechische Republik 2002). Der Besitz persönlicher Gegenstände ist stark eingeschränkt, Dekoration des Haftraums - wozu z. B. auch Bücherregale zählen können 
- ist nicht erlaubt (vgl. die Berichte über Estland 1997, die Tschechische Republik 2006). Häufig ist die Abteilung nicht nur nach außen besonders gesichert, sondern auch nach innen: Die Gefangenen werden zumindest mit Handschellen gefesselt, sobald sie den Haftraum verlassen (vgl. die Berichte über Estland 1997, Lettland 2002, Ungarn 2005, Slowakei 2005, Tschechische Republik 2002, 2006). Das gilt auch für Arztbesuche, bei denen dann auch Aufsichtspersonal anwesend ist (vgl. die Berichte über Lettland 2002, Slowakei 2005, Tschechische Republik 2002, 2006). Die grundsätzliche Einschätzung als gefährlich kann aber auch für die Rahmenbedingungen bei Besuchen eine Rolle spielen, die dann in einem Raum mit Trennscheibe stattfinden (vgl. die Berichte über die Slowakei 2005, Tschechische Republik 2002, Ungarn 2007). Festzuhalten ist, dass es sich hier nicht um die Bedingungen handelt, unter denen Arrest vollzogen wird, sondern um das normale Regime.

Ein weiterer Kritikpunkt des CPT, der sehr häufig genannt wird, ist der Mangel an sinnvoller Beschäftigung. Dieser Mangel ist dem obenbeschriebenen Regime inhärent (vgl. die Berichte über Bulgarien 1999, Italien 2004, Lettland 1999, 2002, Litauen 2004, Slowakei 1995, 2005, Tschechische Republik 2002). Aber auch wenn die materiellen Bedingungen besser sind und das Regime - jedenfalls nach innen - offener ist, gibt es oft nicht genügend Arbeits- und Ausbildungsplätze, Behandlungsmaßnahmen, Angebote an Sport und anderen Freizeitaktivitäten (vgl. die Berichte über Belgien 2005, Deutschland 1996, Finnland 1998, Frankreich 2003, Griechenland 1997, Niederländische Antillen 1994, Zypern 2000). Auch der Mangel an Entlassungsvorbereitungen fällt dem Komitee immer wieder auf (vgl. die Berichte über Frankreich 2000, 2003). Dies sind Probleme, die in vielen Ländern den gesamten Strafvollzug betreffen, allerdings fällt dieser Mangel immer mehr ins Gewicht, je länger ein Gefangener im Strafvollzug bleibt. Besonders augenfällig wird der Bedarf an besonders auf Langzeitinhaftierte zugeschnittenen Angeboten bei unbegrenzten Sanktionen. Dazu zählt die lebenslange Freiheitsstrafe ohne die Möglichkeit der Strafrestaussetzung, wie sie in einigen Staaten nach deren Beitritt zum Europarat als Ersatz für die Todesstrafe eingeführt wurde, aber auch z. B. die Sicherungsverwahrung, bei der die Entlassungschancen eher schlecht sind (2004: Beendigung in 26 Fällen, davon in 15 Entlassung in die Freiheit, gleichzeitig 65 neue Anordnungen; Kröniger 2006, S. 16; Statistisches Bundesamt 2006, Tab. 5.1). Selbst wenn die materiellen Bedingungen sehr gut sind und es grundsätzlich die Möglichkeit gibt, sich sinnvoll zu betätigen, besteht die Gefahr, dass Inhaftierte das Angebot nicht wahrnehmen wollen, weil sie darin keinen Sinn sehen, oder es nicht können (vgl. den Bericht über Deutschland 2005, S. 41 f.; s. auch die Empfehlung im Bericht zum Besuch in Luxemburg 2003, S. 46, für eine Gruppe Lebenslanger psychologische Betreuung einzuführen, und den Bericht über Ungarn 2005, S. 32 f.). Außerdem stellt das CPT häufig fest, dass es für Langstrafer keine Vollzugsplanung gibt (vgl. die Berichte über Finnland 1998, Tschechische Republik 2006).

Neben diesen größeren Problemkreisen kommt es vor, dass der Kontakt zur Familie und $\mathrm{zu}$ Bekannten dadurch erschwert wird, dass alle Langstrafer in eine zentrale Anstalt kommen und nicht heimatnah untergebracht werden oder dass Besuche nur während der üblichen Arbeitszeit möglich sind, so dass Besucher gezwungen sind, Urlaub zu nehmen. Bei der Zuteilung von Arbeitsplätzen werden neben den Gefangenen mit Unterhaltspflichten diejenigen bevorzugt, die kurz vor der Entlassung stehen, was dazu führt, dass Gefangene mit langen Strafen sehr lange arbeitslos bleiben.

Bei der Bewertung der Befunde des Komitees anlässlich seiner Besuche ist zu bedenken, dass es sich hier um Stichproben handelt und nicht um eine systematische Bestandsaufnahme des Strafvollzugs in Europa zu einem bestimmten Zeitpunkt. Außerdem wurden hier Berichte über schlechte Zustände zusammengefasst. Ein Beispiel für gute Bedingungen in einer Einrichtung für Langzeitinhaftierte enthält der Bericht über den Besuch in Dänemark 1996, als das CPT die Anstalt in Herstedvester besuchte.

Zudem hat sich die Lage seit den 1990er Jahren in vielen Ländern bzw. in vielen der immer wieder besuchten Vollzugseinrichtungen zumindest hinsichtlich der materiellen Bedingungen erheblich verbessert. Als Beispiel sollen hier die Berichte über die Abteilung für zu lebenslanger Freiheitsstrafe ohne Bewährungsmöglichkeit Verurteilte in Szeged (Ungarn) 2005 und 2007 dienen. Die materiellen Bedingungen wurden hier erheblich verbessert. Allerdings war das Regime - entgegen den ursprünglichen Plänen für diese Abteilung - nach wie vor sehr streng und schränkte soziale Kontakte stark ein (vgl. den Bericht über den Besuch in Ungarn 2007, S. 11 ff.).

\section{Ausblick}

Es besteht ein Defizit an international vergleichender Forschung in Bezug auf Lebensbe- dingungen und psychische Gesundheit von Gefangenen mit langen Strafen in Europa. Auch die Berichte des CPT ergeben kein vollständiges Bild der Lebens- und Haftbedingungen. Zwar werden bei jedem Besuch die gleichen Kriterien angelegt, allerdings wird jeweils nur die Situation in einer bestimmten Anstalt oder einer bestimmten Abteilung zu einer bestimmten Zeit begutachtet. Allerdings ist eine Bestandsaufnahme der Lebensbedingungen im Strafvollzug auch nicht die Aufgabe des CPT, sondern es soll die Einhaltung von Menschenrechtsstandards beobachten.

Der Versuch, international vergleichbare Daten in verschiedenen europäischen Staaten zusammenzutragen, ist bisher nur vom Lehrstuhl für Kriminologie der Universität Greifswald unternommen worden. Gemeinsam mit Partnern im europäischen Ausland wurden in zwei Forschungsprojekten die Lebens- und Haftbedingungen im Hinblick auf Menschenrechtsstandards des Europarats im geschlossenen Männervollzug und im Frauenvollzug untersucht. Am Projekt zum Männervollzug waren neben Deutschland Estland, Finnland, Lettland, Litauen, Polen, Russland und Schweden beteiligt, das Projekt zum Frauenvollzug wurde in Dänemark, Deutschland, Spanien, Griechenland, Kroatien, Litauen, Polen, Russland und Slowenien durchgeführt (Dünkel/Kestermann/Zolondek 2006). Derzeit läuft ein drittes Projekt zu den Lebensbedingungen im Vollzug langer Freiheitsstrafen, bei dem erstmals auch Daten zu psychiatrischen Erkrankungen erhoben werden und an dem neben Deutschland Belgien, Dänemark, Spanien, Finnland, Frankreich, Großbritannien, Kroatien, Litauen, Polen und Schweden beteiligt sind. Allen drei Projekten liegt ein vergleichbarer Ansatz zugrunde, so dass es möglich sein wird, die Daten untereinander zu vergleichen.

Dr. med. Manuela Dudeck ist Fachärztin für Nervenheilkunde, Tiefenpsychologisch fundierte Psychotherapie und Forensische Psychiatrie an der Klinik und Poliklinik für Psychiatrie und Psychotherapie der Ernst-Moritz-Arndt Universität Greifswald am Hanseklinikum Stralsund.

Dr. Kirstin Drenkhahn ist Assistentin am Lehrstuhl für Kriminologie der Universität Greifswald

\section{Literaturangaben}

Andersen, H. S. (2004): Mental health in prison populations. A review with special emphasis on a study of Danish prisoners on remand. Acta Psychiatrica Scandinavica 424, S. 5-59.

Andersen, H.S., Sestoft, D., Lillebaek, T., Gabrielsen, G., Kramp, P. (1996): Prevalence of ICD-10 Psychiatric Morbidity in Random Samples of Prisoners on Re- 
mand. International Journal of Law and Psychiatry 19, S. 61-74.

Arbeitskreis Sozialtherapeutische Anstalten im Justizvollzug e. V. (2001): Mindestanforderungen an Organisationsform, räumliche Voraussetzungen und Personalausstattung Sozialtherapeutischer Einrichtungen. Informationsschrift des Arbeitskreises Sozialtherapeutische Anstalten im Justizvollzug e. V. 1/2001, S. 6-9.

Birmingham, L., Mason, D., Grubin, D. (1996): Prevalence of mental disorder in remand prisoners: consecutive case study. The British Journal of Psychiatry 313, S. 1521-1524.

Birmingham, L., Wilson, S., Adshead, G. (2006): Prison medicine: ethics and equivalence. The British Journal of Psychiatry 188, S. 4-6.

Blocher, D., Henkel, K., Ziegler, M., Rösler, M. (2001): Prevalence of psychopathological distress in a male prison population. Recht \& Psychiatrie 19, S. 136-140.

Brooke, D., Taylor, C., Gunn, J., Maden, A. (1996): Point prevalence of mental disorder in unconvicted male prisoners in England and Wales. The British Journal of Psychiatry 313, S. 1524-1527.

Bussmann, K.-D., Seifert, S. (2002): Kriminologische Evaluation der Sozialtherapeutischen Anstalt Halle (Saale). 1. Zwischenbericht. Manuskript. Halle.

Bussmann, K.-D., Seifert, S., Richter, K., Kranhold, A. (2004): Kriminologische Evaluation der Sozialtherapeutischen Anstalt Halle (Saale). 2. Zwischenbericht. Manuskript. Halle.

Carrá, G., Giacobone, C., Pozzi, F., Alecci, P., Barale, F. (2004): Prevalence of mental disorder and related treatments in a local jail: a 20-month consecutive case study. Epidemiologia e psichiatria sociale 13, S. 47-54.

Cooke, D.J., Mitchie, C. (1996). Suicide in scottish prisons: A methodological note. Legal and Criminological Psychology 1, S. 287-293.

Dudeck, M., Freyberger, H.J. (in press): Psychische Folgeschäden bei Delinquenzopfern. In: Leygraf, N., Saß, H., Kröber, H.L. (Hrsg.): Handbuch der Forensischen Psychiatrie. Bd.3 Forensische Psychiatrie im Privatrecht und öffentlichen Recht. Steinkopff - Verlag Darmstadt.

Dudeck, M., Spitzer, C., Stopsack, M., Freyberger, H.J., Barnow, S. (2007): Forensic Inpatient Male Sexual Offenders: The Impact of Personality Disorder and Childhood Sexual Abuse. Journal of Forensic Psychiatry and Psychology 18(4),494-506.

Dünkel, F. (1980): Legalbewährung nach sozialtherapeutischer Behandlung. Berlin.

Dünkel, F., Kestermann, C., Zolondek, J. (2006): Reader: Internationale Studie zum Frauenstrafvollzug - Bestandsaufnahme, Bedarfsanalyse und „,best practice“. Greifswald. Internetpublikation, http://www. rsf.uni-greifswald.de/duenkel/publikationen/internet/frauenvollzug.html.

Europäisches Komitee zur Verhütung von Folter und unmenschlicher oder erniedrigender Behandlung oder Strafe (CPT, 2003): Verhütung von Misshandlungen. CPT/Inf/E (2002) 3, http://www.cpt.coe. int/lang/deu/deu-booklet-p.pdf.

Europäisches Komitee zur Verhütung von Folter und unmenschlicher oder erniedrigender Behandlung oder Strafe (CPT, 2004): Die Standards des CPT. CPT/ Inf/E (2002) 1 - Rev. 2004, http://www.cpt.coe. int/lang/deu/deu-standards-p.pdf.
Europarat (Hrsg., 2001): SPACE I - Survey 2000. Bearbeitet von P. V. Tournier. Strasbourg.

Europarat (Hrsg., 2002): SPACE I - Survey 2001. Bearbeitet von P. V. Tournier. Strasbourg.

Europarat (Hrsg., 2003): SPACE I - Survey 2002. Bearbeitet von M. F. Aebi. Strasbourg.

Europarat (Hrsg., 2004): SPACE I - Survey 2003. Bearbeitet von M. F. Aebi. Strasbourg.

Europarat (Hrsg., 2005): SPACE I - Survey 2004. Bearbeitet von M. F. Aebi. Strasbourg.

Europarat (Hrsg., 2007): SPACE I - Survey 2005. Bearbeitet von M. F. Aebi und N. Stadnic. Strasbourg.

Falissard, B., Loze, J.Y., Gasquet, I., Duburc, A., de Beaurepaire, Ch., Fagnani, F., Rouillon, F. (2006): Prevalence of mental disorders in French prison for men. BMC Psychiatry 6, S. 1-6.

Farrell, M., Boys, A. (2002): Psychosis and drug dependence: results from a national survey of prisoners. The British Journal of Psychiatry 181, S. 393-398.

Fazel, S., Hope, T., O’Donnell, I., Jacoby, R. (2001): Hidden psychiatric morbidity in elderly prisoners. The British Journal of Psychiatry 179, S. 535-539.

Fotiadou, M., Livaditis, M., Manou, E., Kaniotou, E., Xenitidis, K. (2006): Prevalence of mental disorders and deliberate self-harm in Greek male prisoners. International Journal of Law and Psychiatry 29, S. 68-73.

Harsch, S., Bergk, J.E., Steinert, T., Keller, F., Jockusch, U. (2006): Prevalence of mental disorders among sexual offenders in forensic psychiatry and prison. International Journal of Law and Psychiatry 29, S. 443-449.

Hinsch, R., Pfingsten, U. (2007): Gruppentraining sozialer Kompetenzen. Weinheim.

von Hofer, H. (2004): Die Entwicklung der Gefangenenraten in achtzehn europäischen Ländern, 1983-2002: ein Ausdruck für neue Straflust. Kriminologisches Journal, 8. Beiheft, S. 196-205.

Jacobs, S., Reinhold, B. (2004): Psychiatric Disorders of Adolescents in Prison. Recht \& Psychiatrie 22 (3), S. 142-146.

Joukamaa, M. (1995): Psychiatric morbidity among Finnish prisoners with special reference to sociodemographic factors: results of the Health Survey of Finnish Prisoners (Wattu Project). Forensic Science International 73, S. 85-91.

Kanfer, F.H., Reinecker, H., Schmelzer, D. (2004): Selbstmanagement-Therapie. 4. Auflage, Berlin.

Konrad, N. (2000): Psychiatrie in Haft, Gefangenschaft und Gefängnis. In: Helmchen, H., Henn, F., Lauter, H., Sartorius, N. (2000): Psychiatrie der Gegenwart, Band 3. Berlin, S. 555-576.

Konrad, N. (2004): Prävalenz psychischer Störungen bei Verbüßern einer Ersatzfreiheitsstrafe. Recht \& Psychiatrie 22, S. 147-150.

Kröniger, S. (2006): Lebenslange Freiheitsstrafe, Sicherungsverwahrung und Unterbringung in einem psychiatrischen Krankenhaus - Dauer und Gründe der Beendigung. Wiesbaden.

Langeveld, H., Melhus, H. (2004): Are psychiatric disorders identified and treated by inprison health services? Tidsskr Nor Laegeforen 124, S. 294.

Liebling, A. (2004): Prisons and their moral performance. Oxford, New York.
Missoni, L., Friedrich, M., Konrad, N. (2003): Psychi(atri)sche Störungen bei Untersuchungsgefangenen. ZfStrVo 52, S. 323-333.

Ortmann, R. (2002): Sozialtherapie im Strafvollzug. Freiburg i. Br.

Rasmussen, K., Almvik, R., Levander, S. (2001): Attention Deficit Hyperactivity Disorder, Reading Disability, and Personality Disorders in a Prison Population. The Journal of the American Academy of Psychiatry and the Law 29, S. 186-193.

Roesch, R., Ogloff, J.R.P., Eaves, D. (1995): Mental health research in the criminal justice system: the need for common approaches and international perspectives. International Journal of Law and Psychiatry 18, S. 1-14.

Rösler, M., Retz, W., Retz-Junginger, P., Hengesch, G., Schneider, M., Supprian, T., Schwitzgebel, P., Pinhard, K., Dovi-Akue, N., Wender, P., Thome, J. (2004): Prevalence of attention deficit-/hyperactivity disorder (ADHD) and comorbid disorders in young male prison inmates. European Archives of Psychiatry and Clinical Neuroscience 254, S. 365-371.

Schafer, G., Schubert, W., Bartels, M., Foerster, K. (2004): Liaison Psychiatry in the Prison of Rottenburg (Germany). Psychiatrische Praxis 31, S. 4-10.

von Schönfeld, C.E., Schneider, F., Schröder, T., Widmann, B., Bothof, U., Driessen, M. (2006): Prävalenz psychischer Störungen, Psychopathologie und Behandlungsbedarf bei weiblichen und männlichen Gefangenen. Der Nervenarzt 77, S. 830841.

Sparks, R., Bottoms, A., Hay, W. (1996): Prisons and the problem of order. Oxford.

Specht, F. (2000): Arbeitskreis Sozialtherapeutischer Anstalten im Justizvollzug. In: Egg, R. (Hrsg.): Behandlung von Sexualstraftätern im Justizvollzug. Wiesbaden, S. 19-24.

Spitzer, C., Dudeck, M., Liß, H., Orlob, S., Gillner, M., Freyberger, H.J. (2001): Posttraumatic stress disorder in forensic patients. Journal of Forensic Psychiatry, April 2007, 63-77.

Statistisches Bundesamt (2006): Strafverfolgung 2004. Fachserie 10, Reihe 3. Wiesbaden.

Vreugdenhil, C., Doreleijers, T.A.H., Vermeiren, R., Wouters, L.F.J.M., van den Brink, W. (2004): Psychiatric Disorders in a Representative Sample of Incarcerated Boys in The Netherlands. Journal of the American Academy of Child \& Adolescent Psychiatry 43, S. 97-104.

Walmsley, R. (2006): World Prison Population List, 7 th edition. Home Office Research, Development and Statistics Directorate. London.

Watzke, S., Ullrich, S., Marneros, A. (2006): Genderand violence-related prevalence of mental disorders in prisoners. European Archives of Psychiatry and Clinical Neuroscience 256, S. 414-421.

Wilson, S. (2004): The principle of equivalence and the future of mental health care in prison. The British Journal of Psychiatry 184, S. 5-7.

Die Berichte über die Besuche des CPT und die Stellungnahmen der Regierungen sind unter http://www.cpt.coe.int/en/states.htm nach Staaten sortiert zu finden. 